International Journal of Pure and Applied Mathematics

Volume 99 No. 1 2015, 51-64

ISSN: 1311-8080 (printed version); ISSN: 1314-3395 (on-line version)

url: http://www.ijpam.eu

doi: http://dx.doi.org/10.12732/ijpam.v99i1.5

ijpam.eu

\title{
GENERALIZATIONS OF THE HERMITE-HADAMARD TYPE INEQUALITIES FOR HARMONICALLY UASI-CONVEX FUNCTIONS
}

\author{
Jaekeun Park \\ Department of Mathematics \\ Hanseo University \\ Seosan, Chungnam, 356-706, KOREA
}

\begin{abstract}
In this paper, some new results related to the right-hand side of the Hermite-Hadamard type inequality for the class of functions whose derivatives at certain powers are harmonically quasi-convex functions are obtained.
\end{abstract}

AMS Subject Classification: 26A51, 26D15

Key Words: Hermite-Hadamard type inequality, Simpson type inequality, Hölder's inequality, harmonically convexity

\section{Introduction}

Many inequalities have been established for convex functions but the most famous is the Hermite-Hadamard's inequality, due to its rich geometrical significance and applications, which is stated as follows: Let $f: I \subseteq R \rightarrow R$ be

Received: June 16, 2014

(c) 2015 Academic Publications, Ltd. url: www.acadpubl.eu 
a convex function and $a, b \in I$ with $a<b$. Then following double inequalities hold:

$$
\left(\frac{a+b}{2}\right) \leq \frac{1}{b-a} \int_{a}^{b} f(x) \mathrm{d} x \leq \frac{f(a)+f(b)}{2}
$$

Hermite-Hadamard's inequalities for convex, $(\alpha, m)$-convex, $G A$-convex and geometric convex functions have received renewed attention in recent years and a remarkable variety of refinements and generalizations for them has been found in $[1,2,3,7,8,9,10,11,13,14]$ and references therein.

Let us recall some definitions of several kinds of convex functions:

Definition 1. Let $I$ be an interval in $R$. Then $f: I \rightarrow R$ is said to be convex on $I$ if the inequality

$$
f(t x+(1-t) y) \leq t f(x)+(1-t) f(y)
$$

holds, for all $x, y \in I$ and $t \in[0,1]$.

Definition 2. Let $I$ be an interval in $R_{+}=(0, \infty)$. A function $f: I \rightarrow R$ is said to be harmonically convex on $I$ if the inequality

$$
f\left(\frac{x y}{t x+(1-t) y}\right) \leq t f(y)+(1-t) f(x)
$$

holds, for all $x, y \in I$ and $t \in[0,1]$. If the inequality in (2) is reversed, then $f$ is said to be harmonically concave.

In [4], Imdat Işcan established the following result of the Hermite-Hadamard type for harmonically convex functions:

Theorem 1.1. Let $f: I \subseteq R_{+}=(0, \infty) \rightarrow R$ be a harmonically convex function on an interval $I$ and $f \in L[a, b]$, where $a, b \in I$ with $a<b$.

$$
f\left(\frac{2 a b}{a+b}\right) \leq \frac{a b}{b-a} \int_{a}^{b} \frac{f(x)}{x^{2}} \mathrm{~d} x \leq \frac{f(a)+f(b)}{2}
$$

Also, in [4, 5, 6], Imdat Işcan established some new Hermite-Hadamard type and Ostrowski type inequalities, which estimate the difference between the middle and the rightmost terms in (3), for harmonically convex functions:

Theorem 1.2. Let $f: I \subseteq R_{+}=(0, \infty) \rightarrow R$ be a differentiable function on the interior $I^{0}$ of an interval $I$ in $R_{+}=(0, \infty)$ and $f^{\prime} \in L[a, b]$, where $a, b \in I$ 
with $a<b$. If $\left|f^{\prime}\right|^{q}$ is harmonically convex function on $[a, b]$ for $q \geq 1$, then the following inequality holds:

$$
\begin{aligned}
& \left|\frac{f(a)+f(b)}{2}-\frac{a b}{b-a} \int_{a}^{b} \frac{f(x)}{x^{2}} \mathrm{~d} x\right| \\
& \leq \frac{a b(b-a)}{2} \lambda_{1}^{1-\frac{1}{q}}\left[\lambda_{2}\left|f^{\prime}(a)\right|^{q}+\lambda_{3}\left|f^{\prime}(b)\right|^{q}\right]^{\frac{1}{q}},
\end{aligned}
$$

where

$$
\begin{aligned}
\lambda_{1} & =\frac{1}{a b}-\frac{2}{(b-a)^{2}} \ln \left(\frac{(a+b)^{2}}{4 a b}\right), \\
\lambda_{2} & =-\frac{1}{b(b-a)}+\frac{3 a+b}{(b-a)^{3}} \ln \left(\frac{(a+b)^{2}}{4 a b}\right), \\
\lambda_{3} & =\frac{1}{a(b-a)}-\frac{3 b+a}{(b-a)^{3}} \ln \left(\frac{(a+b)^{2}}{4 a b}\right) \\
& =\lambda_{1}-\lambda_{2} .
\end{aligned}
$$

In [15], Zhang et. al defined the harmonically quasi-convex function and supplied several properties of this kind of functions.

Definition 3. Let $I$ be an interval in $R_{+}=(0, \infty)$. A function $f: I \rightarrow R$ is said to be harmonically quasi-convex on $I$ if the inequality

$$
f\left(\frac{x y}{t x+(1-t) y}\right) \leq \sup \{f(x), f(y)\}
$$

holds, for all $x, y \in I$ and $t \in[0,1]$. If the inequality in (2) is reversed, then $f$ is said to be harmonically quasi-concave.

In this article we consider the following special functions:

Definition 4. The hypergeometric function ${ }_{2} F_{1}[a, b, c, x]$ is defined for $|x|<1$ by the power series

$$
{ }_{2} F_{1}[a, b, c, x]=\Sigma_{n=0}^{\infty} \frac{(a)_{n}(b)_{n}}{(c)_{n}} \frac{x^{n}}{n !} .
$$

Here $(q)_{n}$ is the Pochhammer symbol, which is defined by

$$
(q)_{n}= \begin{cases}1, & n=0 \\ q(q+1) \cdots(q+n-1), & n>0 .\end{cases}
$$


In this paper, we give some new Hermite-Hadamard type inequalities, which gives an upper bound for the approximation of the integral average $\frac{1}{b-a} \int_{a}^{b} f(u) \mathrm{d} u$ by the value $\frac{f(a)+f(b)}{2}$, that is, estimate the difference between the middle and the rightmost terms in (1), for harmonically $s$-convex functions in the second sense by setting up an integral identity for differentiable functions.

\section{Main Results}

In order to find some new inequalities of Hermite-Hadamard-like type inequalities connected with the rightmost and and middle parts of (1) for functions whose derivatives are harmonically $s$-convex in the second sense, we need the following lemma [12]:

Lemma 2.1. Let $f: I \subseteq R_{+}=(0, \infty) \rightarrow R$ be a differentiable function on the interior $I^{0}$ of an interval $I$ such that $f^{\prime} \in L([a, b])$, where $a, b \in I$ with $a<b$. Then the following identity

$$
\begin{aligned}
& \frac{r f(a)+f(b)}{r+1}-\frac{1}{b-a} \int_{a}^{b} f(x) \mathrm{d} x \\
& =\frac{a b(a-b)}{r+1} \int_{0}^{1} \frac{1-(r+1) t}{A_{t}^{2}(a, b)} f^{\prime}\left(\frac{a b}{A_{t}(a, b)}\right) \mathrm{d} t
\end{aligned}
$$

holds for $r \in[0,1]$, where $A_{t}(a, b)=(1-t) a+t b$.

Proof By the integration by parts, we have

$$
\begin{aligned}
& \int_{0}^{1} \frac{1-(r+1) t}{A_{t}^{2}(a, b)} f^{\prime}\left(\frac{a b}{A_{t}(a, b)}\right) \mathrm{d} t \\
& =\frac{1}{a b(b-a)}\left[r f(a)+f(b)-(r+1) \int_{0}^{1} f\left(\frac{a b}{A_{t}(a, b)}\right) \mathrm{d} t\right] \\
& =\frac{1}{a b(b-a)}\left[r f(a)+f(b)-(r+1) \frac{a b}{b-a} \int_{a}^{b} \frac{f(x)}{x^{2}} \mathrm{~d} x\right],
\end{aligned}
$$

which implies that the identity (6) holds.

Now we turn our attention to establish the Hermite-Hadamard type inequalities, which estimate the difference between the middle and the leftmost terms in (1), for harmonically quasi-convex functions in the second sense by using the above lemma. 
Theorem 2.1. Let $f: I \subseteq R_{+}=(0, \infty) \rightarrow R$ be a differentiable function on $I^{0}$, the interior of an interval $I$, such that $f^{\prime} \in L([a, b])$, where $a, b \in I$ with $a<b$. If $\left|f^{\prime}\right|$ is harmonically quasi-convex on $[a, b]$, then for all $t \in[0,1]$ the following inequality holds:

$$
\begin{aligned}
& \left|\frac{r f(a)+f(b)}{r+1}-\frac{a b}{b-a} \int_{a}^{b} \frac{f(x)}{x^{2}} \mathrm{~d} x\right| \\
& \leq\left\{\frac{b-r a}{r+1}+\frac{a b}{b-a} \ln \left[\frac{a b(r+1)^{2}}{(r a+b)^{2}}\right]\right\} \sup \left\{\left|f^{\prime}(a)\right|,\left|f^{\prime}(b)\right|\right\} .
\end{aligned}
$$

Proof From Lemma 1, we have

$$
\begin{aligned}
& \left|\frac{r f(a)+f(b)}{r+1}-\frac{a b}{b-a} \int_{a}^{b} \frac{f(x)}{x^{2}} \mathrm{~d} x\right| \\
& \leq \frac{a b(b-a)}{r+1} \int_{0}^{1} \frac{|1-(r+1) t|}{A_{t}^{2}(a, b)}\left|f^{\prime}\left(\frac{a b}{A_{t}(a, b)}\right)\right| \mathrm{d} t \\
& =\frac{a b(b-a)}{r+1}\left[\int_{0}^{\frac{1}{r+1}} \frac{1-(r+1) t}{A_{t}^{2}(a, b)}\left|f^{\prime}\left(\frac{a b}{A_{t}(a, b)}\right)\right| \mathrm{d} t\right. \\
& \left.\quad+\int_{\frac{1}{r+1}}^{1} \frac{(r+1) t-1}{A_{t}^{2}(a, b)}\left|f^{\prime}\left(\frac{a b}{A_{t}(a, b)}\right)\right| \mathrm{d} t\right] .
\end{aligned}
$$

Since $\left|f^{\prime}\right|^{q}$ is harmonically quasi-convex on $[a, b]$, we have

$$
\begin{aligned}
& \left|\frac{r f(a)+f(b)}{r+1}-\frac{a b}{b-a} \int_{a}^{b} \frac{f(x)}{x^{2}} \mathrm{~d} x\right| \\
& \leq \frac{a b(b-a)}{r+1}\left\{\int_{0}^{\frac{1}{r+1}} \frac{1-(r+1) t}{A_{t}^{2}(a, b)} \mathrm{d} t+\int_{\frac{1}{r+1}}^{1} \frac{(r+1) t-1}{A_{t}^{2}(a, b)} \mathrm{d} t\right\} \\
& \quad \times \sup \left\{\left|f^{\prime}(a)\right|,\left|f^{\prime}(b)\right|\right\} \\
& =\left\{\frac{b-r a}{r+1}+\frac{a b}{b-a} \ln \left[\frac{a b(r+1)^{2}}{(r a+b)^{2}}\right]\right\} \sup \left\{\left|f^{\prime}(a)\right|,\left|f^{\prime}(b)\right|\right\},
\end{aligned}
$$

where we have used the facts that

$$
\begin{aligned}
& \text { (i) } \int_{0}^{\frac{1}{r+1}} \frac{1-(r+1) t}{A_{t}^{2}(a, b)} \mathrm{d} t=\frac{1}{a(b-a)}+\frac{r+1}{(b-a)^{2}} \ln \left[\frac{a(r+1)}{r a+b}\right] \\
& \text { (ii) } \int_{\frac{1}{r+1}}^{1} \frac{(r+1) t-1}{A_{t}^{2}(a, b)} \mathrm{d} t=-\frac{r}{b(b-a)}+\frac{r+1}{(b-a)^{2}} \ln \left[\frac{b(r+1)}{r a+b}\right] .
\end{aligned}
$$


Therefore, we can deduce the following results:

Corollary 2.1. Let $f: I \subseteq R_{+}=(0, \infty) \rightarrow R$ be a differentiable function on $I^{0}$, the interior of an interval $I$, such that $f^{\prime} \in L([a, b])$, where $a, b \in I$ with $a<b$. Assume $\left|f^{\prime}\right|$ is harmonically quasi-convex on $[a, b]$.

(1) If $r=1$ in (7), then the following inequality holds:

$$
\begin{aligned}
& \left|\frac{f(a)+f(b)}{2}-\frac{a b}{b-a} \int_{a}^{b} \frac{f(x)}{x^{2}} \mathrm{~d} x\right| \\
& \leq\left\{\frac{b-a}{2}+\frac{a b}{b-a} \ln \left[\frac{4 a b}{(a+b)^{2}}\right]\right\} \sup \left\{\left|f^{\prime}(a)\right|,\left|f^{\prime}(b)\right|\right\} .
\end{aligned}
$$

(2) If $r=0$ in (7), then the following inequality holds:

$$
\begin{aligned}
& \left|f(b)-\frac{a b}{b-a} \int_{a}^{b} \frac{f(x)}{x^{2}} \mathrm{~d} x\right| \\
& \leq\left\{b+\frac{a b}{b-a} \ln \left[\frac{a}{b}\right]\right\} \sup \left\{\left|f^{\prime}(a)\right|,\left|f^{\prime}(b)\right|\right\} .
\end{aligned}
$$

Theorem 2.2. Let $f: I \subseteq R_{+}=(0, \infty) \rightarrow R$ be a differentiable function on $I^{0}$, the interior of an interval $I$, such that $f^{\prime} \in L([a, b])$, where $a, b \in I$ with $a<b$. If $\left|f^{\prime}\right|^{q}$ is harmonically quasi-convex on $[a, b]$ for $q \geq 1$, then fot all $x \in[a, b]$ the following inequality holds:

$$
\begin{aligned}
& \left|\frac{r f(a)+f(b)}{r+1}-\frac{a b}{b-a} \int_{a}^{b} \frac{f(x)}{x^{2}} \mathrm{~d} x\right| \\
& \leq \frac{a b(b-a)}{(r+1)^{1+\frac{1}{p}}}\left\{\mu_{21}^{\frac{1}{q}}(a, b, r, q)+r^{\frac{1}{p}} \mu_{22}^{\frac{1}{q}}(a, b, r, q)\right\} \\
& \quad \times\left(\sup \left\{\left|f^{\prime}(a)\right|^{q},\left|f^{\prime}(b)\right|^{q}\right\}\right)^{\frac{1}{q}},
\end{aligned}
$$

where

$$
\begin{array}{ll}
\mu_{21}(a, b, r, q) & =\frac{1}{(1+r)(1+q) a^{2 q}} \quad{ }_{2} F_{1}\left[1,2 q, 2+q,-\frac{b-a}{(1+r) a}\right] \\
\mu_{22}(a, b, r, q) & =\frac{r^{1+q}(1+r)^{2 q-1}}{(1+q)(r a+b)^{2 q}}
\end{array}
$$

Proof From Lemma 1, we have

$$
\left|\frac{r f(a)+f(b)}{r+1}-\frac{a b}{b-a} \int_{a}^{b} \frac{f(x)}{x^{2}} \mathrm{~d} x\right|
$$




$$
\begin{gathered}
\leq \frac{a b(b-a)}{r+1}\left[\int_{0}^{\frac{1}{r+1}} \frac{1-(r+1) t}{A_{t}^{2}(a, b)}\left|f^{\prime}\left(\frac{a b}{A_{t}(a, b)}\right)\right| \mathrm{d} t\right. \\
\left.\quad+\int_{\frac{1}{r+1}}^{1} \frac{(r+1) t-1}{A_{t}^{2}(a, b)}\left|f^{\prime}\left(\frac{a b}{A_{t}(a, b)}\right)\right| \mathrm{d} t\right] .
\end{gathered}
$$

By the harmonically quasi-convexity of $\left|f^{\prime}\right|^{q}$ and using the Hölder integral inequality, we have

$$
\begin{aligned}
\mid & \frac{r f(a)+f(b)}{r+1}-\frac{a b}{b-a} \int_{a}^{b} \frac{f(x)}{x^{2}} \mathrm{~d} x \mid \\
\leq & \frac{a b(b-a)}{r+1}\left[\left(\int_{0}^{\frac{1}{r+1}} 1 \mathrm{~d} t\right)^{\frac{1}{p}}\left(\int_{0}^{\frac{1}{r+1}} \frac{\{1-(r+1) t\}^{q}}{A_{t}^{2 q}(a, b)} \mathrm{d} t\right)^{\frac{1}{q}}\right. \\
& \left.+\left(\int_{\frac{1}{r+1}}^{1} 1 \mathrm{~d} t\right)^{\frac{1}{p}}\left(\int_{\frac{1}{r+1}}^{1} \frac{\{(r+1) t-1\}^{q}}{A_{t}^{2 q}(a, b)} \mathrm{d} t\right)^{\frac{1}{q}}\right] \\
= & \frac{a b(b-a)}{(r+1)^{1+\frac{1}{p}}}\left[\left(\int_{0}^{\frac{1}{r+1}} \frac{\{1-(r+1) t\}^{q}}{A_{t}^{2 q}(a, b)} \mathrm{d} t\right)^{\frac{1}{q}}\right. \\
& \left.+r^{\frac{1}{p}}\left(\int_{\frac{1}{r+1}}^{1} \frac{\{(r+1) t-1\}^{q}}{A_{t}^{2 q}(a, b)} \mathrm{d} t\right)^{\frac{1}{q}}\right]\left(\sup \left\{\left|f^{\prime}(a)\right|^{q},\left|f^{\prime}(b)\right|^{q}\right\}\right)^{\frac{1}{q}} \\
= & \frac{a b(b-a)}{(r+1)^{1+\frac{1}{p}}}\left\{\mu_{21}^{\frac{1}{q}}(a, b, r, q)+r^{\frac{1}{q}} \mu_{22}^{\frac{1}{q}}(a, b, r, q)\right\} \\
& \times\left(\sup \left\{\left|f^{\prime}(a)\right|^{q},\left|f^{\prime}(b)\right|^{q}\right\}\right)^{\frac{1}{q}} \cdot
\end{aligned}
$$

Corollary 2.2. In the inequality (8) in Theorem 2.2, additionally, if $\left|f^{\prime}(x)\right| \leq M$ for $x \in[a, b]$, then the following inequality hold:

$$
\begin{aligned}
& \left|\frac{r f(a)+f(b)}{r+1}-\frac{a b}{b-a} \int_{a}^{b} \frac{f(x)}{x^{2}} \mathrm{~d} x\right| \\
& \leq \frac{a b(b-a) M}{(r+1)^{1+\frac{1}{p}}}\left\{\mu_{21}^{\frac{1}{q}}(a, b, r, q)+r^{\frac{1}{p}} \mu_{22}^{\frac{1}{q}}(a, b, r, q)\right\} .
\end{aligned}
$$

Theorem 2.3. Let $f: I \subseteq R_{+}=(0, \infty) \rightarrow R$ be a differentiable function on $I^{0}$, the interior of an interval $I$, such that $f^{\prime} \in L([a, b])$, where $a, b \in I$ with $a<b$. If $\left|f^{\prime}\right|^{q}$ is harmonically quasi-convex on $[a, b]$ for $q \geq 1$ with $\frac{1}{p}+\frac{1}{q}=1$, 
then for all $x \in[a, b]$ the following inequality holds:

$$
\begin{aligned}
& \left|\frac{r f(a)+f(b)}{r+1}-\frac{a b}{b-a} \int_{a}^{b} \frac{f(x)}{x^{2}} \mathrm{~d} x\right| \\
& \leq \frac{a b(b-a)}{r+1}\left\{\frac{b-r a}{a b(b-a)}+\frac{r+1}{(b-a)^{2}} \ln \left[\frac{a b(r+1)^{2}}{(r a+b)^{2}}\right]\right\} \\
& \quad \times\left(\sup \left\{\left|f^{\prime}(a)\right|^{q},\left|f^{\prime}(b)\right|^{q}\right\}\right)^{\frac{1}{q}} .
\end{aligned}
$$

Proof From Lemma 1, we have

$$
\begin{aligned}
& \left|\frac{r f(a)+f(b)}{r+1}-\frac{a b}{b-a} \int_{a}^{b} \frac{f(x)}{x^{2}} \mathrm{~d} x\right| \\
& \leq \frac{a b(b-a)}{r+1}\left[\int_{0}^{\frac{1}{r+1}} \frac{1-(r+1) t}{A_{t}^{2}(a, b)}\left|f^{\prime}\left(\frac{a b}{A_{t}(a, b)}\right)\right| \mathrm{d} t\right. \\
& \left.\quad+\int_{\frac{1}{r+1}}^{1} \frac{(r+1) t-1}{A_{t}^{2}(a, b)}\left|f^{\prime}\left(\frac{a b}{A_{t}(a, b)}\right)\right| \mathrm{d} t\right] .
\end{aligned}
$$

By the harmonically quasi-convexity of $\left|f^{\prime}\right|^{q}$ and using the Hölder integral inequality, we have

$$
\begin{aligned}
& \left|\frac{r f(a)+f(b)}{r+1}-\frac{a b}{b-a} \int_{a}^{b} \frac{f(x)}{x^{2}} \mathrm{~d} x\right| \\
& \leq \frac{a b(b-a)}{r+1} \\
& \times \quad\left[\left(\int_{0}^{\frac{1}{r+1}} \frac{1-(r+1) t}{A_{t}^{2}(a, b)} \mathrm{d} t\right)^{\frac{1}{p}}\left(\int_{0}^{\frac{1}{r+1}} \frac{1-(r+1) t}{A_{t}^{2}(a, b)} \mathrm{d} t\right)^{\frac{1}{q}}\right. \\
& \left.+\left(\int_{\frac{1}{r+1}}^{1} \frac{(r+1) t-1}{A_{t}^{2}(a, b)} \mathrm{d} t\right)^{\frac{1}{p}}\left(\int_{\frac{1}{r+1}}^{1} \frac{(r+1) t-1}{A_{t}^{2}(a, b)} \mathrm{d} t\right)^{\frac{1}{q}}\right] \\
& \quad \times\left(\sup \left\{\left|f^{\prime}(a)\right|^{q},\left|f^{\prime}(b)\right|^{q}\right\}\right)^{\frac{1}{q}} \\
& =\frac{a b(b-a)}{r+1}\left[\int_{0}^{\frac{1}{r+1}} \frac{1-(r+1) t}{A_{t}^{2}(a, b)} \mathrm{d} t+\int_{\frac{1}{r+1}}^{1} \frac{(r+1) t-1}{A_{t}^{2}(a, b)} \mathrm{d} t\right] \\
& \quad \times\left(\sup \left\{\left|f^{\prime}(a)\right|^{q},\left|f^{\prime}(b)\right|^{q}\right\}\right)^{\frac{1}{q}}
\end{aligned}
$$




$$
\begin{aligned}
=\frac{a b(b-a)}{r+1}\left\{\frac{b-r a}{a b(b-a)}+\frac{r+1}{(b-a)^{2}} \ln \left[\frac{a b(r+1)^{2}}{(r a+b)^{2}}\right]\right\} \\
\times\left(\sup \left\{\left|f^{\prime}(a)\right|^{q},\left|f^{\prime}(b)\right|^{q}\right\}\right)^{\frac{1}{q}},
\end{aligned}
$$

which completes the proof.

Theorem 2.4. Let $f: I \subseteq R_{+}=(0, \infty) \rightarrow R$ be a differentiable function on $I^{0}$, the interior of an interval $I$, such that $f^{\prime} \in L([a, b])$, where $a, b \in I$ with $a<b$. If $\left|f^{\prime}\right|^{q}$ is harmonically quasi-convex on $[a, b]$ for $q>1$ with $\frac{1}{p}+\frac{1}{q}=1$, then for all $x \in[a, b]$ the following inequality holds:

$$
\begin{aligned}
& \left|\frac{r f(a)+f(b)}{r+1}-\frac{a b}{b-a} \int_{a}^{b} \frac{f(x)}{x^{2}} \mathrm{~d} x\right| \\
& \leq a b(b-a)^{\frac{1}{p}}\left(\frac{1}{1+p}\right)^{\frac{1}{p}}\left(\frac{1}{2 q-1}\right)^{\frac{1}{q}} \\
& \times\left[\mu_{41}^{\frac{1}{q}}(a, b, r, q)+r^{1+\frac{1}{p}} \mu_{42}^{\frac{1}{q}}(a, b, r, q)\right] \\
& \quad \times\left(\sup \left\{\left|f^{\prime}(a)\right|^{q},\left|f^{\prime}(b)\right|^{q}\right\}\right)^{\frac{1}{q}},
\end{aligned}
$$

where

$$
\begin{aligned}
& \mu_{41}(a, b, r, q)=\{a(1+r)\}^{1-2 q}-(r a+b)^{1-2 q}, \\
& \mu_{42}(a, b, r, q)=(r a+b)^{1-2 q}-\{b(1+r)\}^{1-2 q} .
\end{aligned}
$$

Proof From Lemma 1 and the the Hölder integral inequality, we have

$$
\begin{aligned}
& \left|\frac{r f(a)+f(b)}{r+1}-\frac{a b}{b-a} \int_{a}^{b} \frac{f(x)}{x^{2}} \mathrm{~d} x\right| \\
& \leq \frac{a b(b-a)}{r+1}\left[\int_{0}^{\frac{1}{r+1}} \frac{1-(r+1) t}{A_{t}^{2}(a, b)}\left|f^{\prime}\left(\frac{a b}{A_{t}(a, b)}\right)\right| \mathrm{d} t\right. \\
& \left.\quad+\int_{\frac{1}{r+1}}^{1} \frac{(r+1) t-1}{A_{t}^{2}(a, b)}\left|f^{\prime}\left(\frac{a b}{A_{t}(a, b)}\right)\right| \mathrm{d} t\right] .
\end{aligned}
$$

By the harmonically quasi-convexity of $\left|f^{\prime}\right|^{q}$ and using the Hölder integral inequality, we have

$$
\begin{aligned}
& \left|\frac{r f(a)+f(b)}{r+1}-\frac{a b}{b-a} \int_{a}^{b} \frac{f(x)}{x^{2}} \mathrm{~d} x\right| \\
& \leq \frac{a b(b-a)}{r+1}
\end{aligned}
$$




$$
\begin{aligned}
\times & {\left[\left(\int_{0}^{\frac{1}{r+1}}\{1-(r+1) t\}^{p} \mathrm{~d} t\right)^{\frac{1}{p}}\left(\int_{0}^{\frac{1}{r+1}} \frac{1}{A_{t}^{2 q}(a, b)} \mathrm{d} t\right)^{\frac{1}{q}}\right.} \\
+ & \left.\left(\int_{\frac{1}{r+1}}^{1}\{(r+1) t-1\}^{p} \mathrm{~d} t\right)^{\frac{1}{p}}\left(\int_{\frac{1}{r+1}}^{1} \frac{1}{A_{t}^{2}(a, b)} \mathrm{d} t\right)^{\frac{1}{q}}\right] \\
& \times\left(\sup \left\{\left|f^{\prime}(a)\right|^{q},\left|f^{\prime}(b)\right|^{q}\right\}\right)^{\frac{1}{q}} \\
= & \frac{a b(b-a)}{r+1}\left[\left(\frac{1}{(1+p)(1+r)}\right)^{\frac{1}{p}}\left(\int_{0}^{\frac{1}{r+1}} \frac{1}{A_{t}^{2 q}(a, b)} \mathrm{d} t\right)^{\frac{1}{q}}\right. \\
+ & \left.\left(\frac{r^{1+p}}{(1+p)(1+r)}\right)^{\frac{1}{p}}\left(\int_{\frac{1}{r+1}}^{1} \frac{1}{A_{t}^{2}(a, b)} \mathrm{d} t\right)^{\frac{1}{q}}\right] \\
\times & \times\left(\sup \left\{\left|f^{\prime}(a)\right|^{q},\left|f^{\prime}(b)\right|^{q}\right\}\right)^{\frac{1}{q}} \\
\leq & a b(b-a)^{\frac{1}{p}}\left(\frac{1}{1+p}\right)^{\frac{1}{p}}\left(\frac{1}{2 q-1}\right)^{\frac{1}{q}} \\
\times & {\left[\mu_{41}^{\frac{1}{q}}(a, b, r, q)+r^{1+\frac{1}{p}} \mu_{42}^{\frac{1}{q}}(a, b, r, q)\right] } \\
& \times\left(\sup \left\{\left|f^{\prime}(a)\right|^{q},\left|f^{\prime}(b)\right|^{q}\right\}\right)^{\frac{1}{q}},
\end{aligned}
$$

where we have used the fact that

$$
\begin{aligned}
& \int_{0}^{\frac{1}{r+1}} \frac{1}{A_{t}^{2 q}(a, b)} \mathrm{d} t=\frac{\{a(1+r)\}^{1-2 q}-(r a+b)^{1-2 q}}{(2 q-1)(1+r)^{1-2 q}(b-a)}, \\
& \int_{\frac{1}{r+1}}^{1} \frac{1}{A_{t}^{2}(a, b)} \mathrm{d} t=\frac{(r a+b)^{1-2 q}-\{b(1+r)\}^{1-2 q}}{(2 q-1)(1+r)^{1-2 q}(b-a)}, \\
& \int_{0}^{\frac{1}{r+1}}(1-(r+1) t)^{p} \mathrm{~d} t=\frac{1}{(1+p)(1+r)}, \\
& \int_{\frac{1}{r+1}}^{1}((r+1) t-1)^{p} \mathrm{~d} t=\frac{1}{(1+p)(1+r)} .
\end{aligned}
$$

Theorem 2.5. Let $f: I \subseteq R_{+}=(0, \infty) \rightarrow R$ be a differentiable function on $I^{0}$, the interior of an interval $I$, such that $f^{\prime} \in L([a, b])$, where $a, b \in I$ with $a<b$. If $\left|f^{\prime}\right|^{q}$ is harmonically quasi-convex on $[a, b]$ for $q>1$ with $\frac{1}{p}+\frac{1}{q}=1$, then for all $x \in[a, b]$ the following inequality holds:

$$
\left|\frac{r f(a)+f(b)}{r+1}-\frac{a b}{b-a} \int_{a}^{b} \frac{f(x)}{x^{2}} \mathrm{~d} x\right|
$$




$$
\begin{aligned}
\leq & a b(b-a)^{\frac{1}{q}}\left(\frac{1}{1+q}\right)^{\frac{1}{q}}\left(\frac{1}{2 p-1}\right)^{\frac{1}{p}} \\
\times & {\left[\mu_{41}^{\frac{1}{p}}(a, b, r, p)+r^{1+\frac{1}{q}} \mu_{42}^{\frac{1}{q}}(a, b, r, p)\right] } \\
& \times\left(\sup \left\{\left|f^{\prime}(a)\right|^{q},\left|f^{\prime}(b)\right|^{q}\right\}\right)^{\frac{1}{q}} .
\end{aligned}
$$

where $\mu_{4 i}(i=1,2)$ are defined in Theorem 2.4 .

Proof From Lemma 1 and the the Hölder integral inequality, we have

$$
\begin{aligned}
& \left|\frac{r f(a)+f(b)}{r+1}-\frac{a b}{b-a} \int_{a}^{b} \frac{f(x)}{x^{2}} \mathrm{~d} x\right| \\
& \leq \frac{a b(b-a)}{r+1}\left[\int_{0}^{\frac{1}{r+1}} \frac{1-(r+1) t}{A_{t}^{2}(a, b)}\left|f^{\prime}\left(\frac{a b}{A_{t}(a, b)}\right)\right| \mathrm{d} t\right. \\
& \left.\quad+\int_{\frac{1}{r+1}}^{1} \frac{(r+1) t-1}{A_{t}^{2}(a, b)}\left|f^{\prime}\left(\frac{a b}{A_{t}(a, b)}\right)\right| \mathrm{d} t\right] .
\end{aligned}
$$

By the harmonically quasi-convexity of $\left|f^{\prime}\right|^{q}$ and using the Hölder integral inequality, we have

$$
\begin{aligned}
& \left|\frac{r f(a)+f(b)}{r+1}-\frac{a b}{b-a} \int_{a}^{b} \frac{f(x)}{x^{2}} \mathrm{~d} x\right| \\
& \leq \frac{a b(b-a)}{r+1}\left(\sup \left\{\left|f^{\prime}(a)\right|^{q},\left|f^{\prime}(b)\right|^{q}\right\}\right)^{\frac{1}{q}} \\
& \times\left[\left(\int_{0}^{\frac{1}{r+1}} \frac{1}{A_{t}^{2 p}(a, b)} \mathrm{d} t\right)^{\frac{1}{p}}\left(\int_{0}^{\frac{1}{r+1}}\{1-(r+1) t\}^{q} \mathrm{~d} t\right)^{\frac{1}{q}}\right. \\
& \left.+\left(\int_{\frac{1}{r+1}}^{1} \frac{1}{A_{t}^{2 p}(a, b)} \mathrm{d} t\right)^{\frac{1}{p}}\right]\left(\int_{\frac{1}{r+1}}^{1}\{(r+1) t-1\}^{q} \mathrm{~d} t\right)^{\frac{1}{q}} \\
& \quad a b(b-a)^{\frac{1}{q}}\left(\frac{1}{1+q}\right)^{\frac{1}{q}}\left(\frac{1}{2 p-1}\right)^{\frac{1}{p}} \\
& \times\left\{\mu_{41}^{\frac{1}{p}}(a, b, r, p)+r^{1+\frac{1}{q}} \mu_{42}^{\frac{1}{p}}(a, b, r, p)\right\} \\
& \times\left(\sup \left\{\left|f^{\prime}(a)\right|^{q},\left|f^{\prime}(b)\right|^{q}\right\}\right)^{\frac{1}{q}} .
\end{aligned}
$$


Theorem 2.6. Let $f: I \subseteq R_{+}=(0, \infty) \rightarrow R$ be a differentiable function on $I^{0}$, the interior of an interval $I$, such that $f^{\prime} \in L([a, b])$, where $a, b \in I$ with $a<b$. If $\left|f^{\prime}\right|^{q}$ is harmonically quasi-convex on $[a, b]$ for $q \geq 1$ with $\frac{1}{p}+\frac{1}{q}=1$, then for all $x \in[a, b]$ the following inequality holds:

$$
\begin{aligned}
& \left|\frac{r f(a)+f(b)}{r+1}-\frac{a b}{b-a} \int_{a}^{b} \frac{f(x)}{x^{2}} \mathrm{~d} x\right| \\
& \leq \frac{a b(b-a)}{(r+1)^{1+\frac{1}{q}}}\left\{\mu_{21}^{\frac{1}{p}}(a, b, r, p)+r^{\frac{1}{q}} \mu_{22}^{\frac{1}{p}}(a, b, r, p)\right\} \\
& \quad \times\left(\sup \left\{\left|f^{\prime}(a)\right|^{q},\left|f^{\prime}(b)\right|^{q}\right\}\right)^{\frac{1}{q}} .
\end{aligned}
$$

Proof From Lemma 1, Hölder integral inequality and the harmonically quasi-convexity of $\left|f^{\prime}\right|^{q}$, we have

$$
\begin{aligned}
& \left|\frac{r f(a)+f(b)}{r+1}-\frac{a b}{b-a} \int_{a}^{b} \frac{f(x)}{x^{2}} \mathrm{~d} x\right| \\
& \leq \frac{a b(b-a)}{r+1}\left[\int_{0}^{\frac{1}{r+1}} \frac{1-(r+1) t}{A_{t}^{2}(a, b)}\left|f^{\prime}\left(\frac{a b}{A_{t}(a, b)}\right)\right| \mathrm{d} t\right. \\
& \left.+\int_{\frac{1}{r+1}}^{1} \frac{(r+1) t-1}{A_{t}^{2}(a, b)}\left|f^{\prime}\left(\frac{a b}{A_{t}(a, b)}\right)\right| \mathrm{d} t\right] \\
& \leq \frac{a b(b-a)}{r+1} \\
& \times\left[\left(\int_{0}^{\frac{1}{r+1}} \frac{\{1-(r+1) t\}^{p}}{A_{t}^{2 p}(a, b)} \mathrm{d} t\right)^{\frac{1}{p}}\left(\int_{0}^{\frac{1}{r+1}}\left|f^{\prime}\left(\frac{a b}{A_{t}(a, b)}\right)\right| \mathrm{d} t\right)^{\frac{1}{q}}\right. \\
& \left.+\left(\int_{\frac{1}{r+1}}^{1} \frac{\{(r+1) t-1\}^{p}}{A_{t}^{2 p}(a, b)} \mathrm{d} t\right)^{\frac{1}{p}}\left(\int_{\frac{1}{r+1}}^{1}\left|f^{\prime}\left(\frac{a b}{A_{t}(a, b)}\right)\right| \mathrm{d} t\right)^{\frac{1}{q}}\right] \\
& \leq \frac{a b(b-a)}{r+1}\left[\mu_{21}^{\frac{1}{p}}(a, b, r, p)\left(\frac{1}{r+1}\right)^{\frac{1}{q}}\left(\sup \left\{\left|f^{\prime}(a)\right|^{q},\left|f^{\prime}(b)\right|^{q}\right\}\right)^{\frac{1}{q}}\right. \\
& \left.+\mu_{22}^{\frac{1}{p}}(a, b, r, p)\left(\frac{r}{r+1}\right)^{\frac{1}{q}}\left(\sup \left\{\left|f^{\prime}(a)\right|^{q},\left|f^{\prime}(b)\right|^{q}\right\}\right)^{\frac{1}{q}}\right] \\
& =\frac{a b(b-a)}{(r+1)^{1+\frac{1}{q}}}\left\{\mu_{21}^{\frac{1}{p}}(a, b, r, p)+r^{\frac{1}{q}} \mu_{22}^{\frac{1}{p}}(a, b, r, p)\right\} \\
& \times\left(\sup \left\{\left|f^{\prime}(a)\right|^{q},\left|f^{\prime}(b)\right|^{q}\right\}\right)^{\frac{1}{q}} \text {. }
\end{aligned}
$$




\section{References}

[1] S. S. Dragomir, Hermite-Hadamard's type inequalities for convex functions of selfadjoint operators in Hilbert spaces, Linear Algebra Appl. 436 (5) (2012), 1503-1515. http://dx.doi.org/10.1016/j.laa2011.08.050.

[2] S. S. Dragomir, Superadditivity and monotonicity of some functionals associated with the Hermite-Hadamard inequality for convex functions in linear spaces, Rocky Mountain J. Math. 42(5) (2012), 1447-1459. http://dx.doi.org/10.1216/RMJ-2012-42-5-1447.

[3] V. N. Huy, N. T. Chung, Some generalizations of the Fejér and HermiteHadamard inequalities in Hölder spaces, J. Appl. Math. Inform. 29(3-4) (2011), 859-868. http://www.kcam.biz.

[4] Imdat Işcan, Hermite-Hadamard type inequalities for harmonically convex functions, arXiv:1303.6089v1[math.CA]25 Mar 2013 (2013).

[5] Imdat Işcan, Hermite-Hadamard and Simpson-like type inequalities for differentiable harmonically convex functions, presented.

[6] Imdat Işcan, Ostrowski type inequalities for harmonically $s$-convex functions, arXiv:submit/0763144[math.CA]19 Jul 2013 (2013).

[7] M. Klaričić Bakula, J. Pečarić, Note on some Hadamard-type inequalities, JIPAM. J. Inequal. Pure Appl. Math. 5(3) (2004), Article 74, 9 pp.

[8] M. Klaričić Bakula, M. E. Özdemir, J. Pečarić, Hadamard type inequalities for $m$-convex and $(\alpha, m)$-convex functions, JIPAM. J. Inequal. Pure Appl. Math. 9(4) (2008), Article 96, 12 pp.

[9] Meihui Qu, Wenjun Liu, J. Park, Some new HermiteHadamard-type inequalities for geometric-arithmetically $s$-convex functions, WSEAS Trans. on Math., 13 (2014), 452-461. http://wseas.org/wseas/cms.action?id $=6850$.

[10] V. G. Miheşan, "A generalization of the convexity" in Seminar on Functional Equations, Approx. and Convex., Cluj-Napoca, Romania, 1993.

[11] M. E. Özdemir, A. Ekinci, A. O. Akdemir, Generalizations of integral inequalities for functions whose second derivatives are convex and $m$-convex, Miskolc Mathematical Notes, 13(2) (2012), 441-457. 
[12] J. Park, Generalization of some Simpson-type inequalities via differentiable $s$-convex mappings in the second sense, Inter. Journ. of Math. and Math Sciences, 2011 (2011), Art ID:493531. http://dx.doi.org/10.1155/2011/493531.

[13] M. Z. Sarikaya, On new Hermite Hadamard Fejér type integral inequalities, Stud. Univ. Babeş-Bolyai Math. 57(3) (2012), 377-386.

[14] T. Y. Zhang, A. P. Ji, F. Qi, Some inequalities of Hermite-Hadamard type for GA-Convex functions with applications to means. Le Matematiche, 68(1) (2013), 229-239. http://dx.doi.org/10.4418/2013.68.1.17.

[15] T. Y. Zhang, A. P. Ji, F. Qi, Integral inequalities of Hermite-Hadamard type for harmonically quasi-convex functions. Proc. Jangjeon Math. Soc., 16(3) (2013), 399-407. 